\title{
Städte zu verkaufen
}

Prozesse der Enteignung und Praktiken der Wiederaneignung in Spanien

\author{
Kike España \\ Beatriz V. Toscano
}

\begin{abstract}
In Spanien zwingen die Wirtschaftszweige Tourismus und Immobilienspekulation zu einer Debatte um die Erosion der Demokratie im Schoß der Europäischen Union, wo sich seit dem Beitritt des Landes die Strategie etabliert hat, dessen Rolle als Produktionsraum für Tourismus zu betonen. Verstärkt seit der Finanzkrise 2008 hat die Tourismusbranche eine gewaltige Transformation erlebt, in deren Zuge Städte zu Hauptreisezielen und -anziehungspunkten geworden sind. Durch bereits vorhandene Raumvermarktungsstrukturen aus dem Immobiliengeschäft haben sich die Möglichkeiten der Tourismusbranche, sich Stadtraum anzueignen, vervielfältigt. Mit der Stadt als Ware werden ganze urbane Zentren in ,Themenparks in Lebensgröße' umgewandelt und die Stadt samt all ihren Lebensressourcen konsumiert. Analysiert wird, inwieweit sich anhand dieser Situation neue Formen eines intra-territorialen Kolonialismus zwischen Touristen bzw. Stadteliten und Bürgern aufzeigen lassen. Ziel des Textes ist es, Möglichkeiten für eine Wiederaneignung dieser Stadträume anzuvisieren, die darauf basieren, Aspekte der Nutzung und des kulturellen Verständnisses dieser Orte neu zu bestimmen.
\end{abstract}

Ersteinreichung: 6. September 2018; Veröffentlichung online: 29. November 2019

An English abstract can be found at the end of the document.

„Il ne faut jamais oublier que la colonisation, avec ses techniques et ses armes politiques et juridiques, a bien sûr transporté des modèles européens sur d'autres continents, mais qu'elle a eu aussi de nombreux effets de retour sur les mécanismes de pouvoir en Occident, sur les appareils, institutions et techniques de pouvoir. Il y a eu toute une série de modèles coloniaux qui ont été rapportés en Occident, et qui a fait que l'Occident a pu pratiquer aussi sur lui-même quelque chose comme une colonisation, un colonialisme interne."

Foucault (2001: 103)

\section{Einleitung}

Der Einband der Romanfassung von Luis García Berlangas Film Willkommen, Mr. Marshall (1953) zeigt ein flüchtiges, doch treffendes prophetisches Porträt der heutigen spanischen Stadt - als Stadt, die sich verkauft. Im Vordergrund zu sehen ist Uncle Sam, der auf etwas reitet, das einer Mischung aus Nikolaussack und Atomrakete ähnelt, und der in einer riesigen Explosion 
Dollarscheine auf die Einwohner_innen von Villa del Río niederregnen lässt. Diese, mit erhobenen Armen tanzend, scheinen zu rufen: „Hier, hier!“, was unweigerlich Erinnerungen an die hungernden Massen in Erwartung der food airdrops weckt. Links im Bild ist ein Einwohner in Volkstracht zu sehen - völlig anachronistisch in den Zeiten des Marshallplans, auf den sich der Film bezieht. Im Hintergrund die schemenhaften Umrisse von Häusern in einem Dorf, das zur Kulisse geworden ist, vor der ein Stück spielt, das ebenso gut ,Spanien` heißen könnte.

Willkommen, Mr. Marshall führt dem Zuschauer die Geschichte von Villa del Río vor; eine während der Nachkriegszeit verarmte und perspektivlose Gemeinschaft, ein Kaff irgendwo in der Mitte Spaniens, dessen raffinierte Einwohner_innen den genialen Einfall hatten, sich als pittoreske ,Spanier_innen“ zu kostümieren (Flamencotrachten, altmodische Banditen und Toreros, wie man sie nur aus den Bildern von Goya kennt). Eine große Summe steht für sie auf dem Spiel; sie werden alles tun, um die nordamerikanischen Gesandten vom Marshallplan mit ihrer volkstümlichen Lebensfreude und Einfachheit für sich zu gewinnen.[1] Nach Ansicht des Bürgermeisters hatte sich das Dorf bei der Umverteilung von Auslandskapital ,schlecht verkauft' - ein unverzeihliches Versäumnis, dem mit der Schaffung einer eigenen Marke, die nunmehr für die Amerikaner ,typisch spanisch“ heißen wird, etwas entgegengesetzt werden soll. Das normale Alltagsgeschehen müssen die Bewohner_innen ruhen lassen, um Statisten in einem fiktiven spanischen Dorf von inszenierter Volkstümlichkeit zu sein, für das sie sich quasi als tableau vivant à la española verkleiden. Dieses Bild wirkt nur nach außen heiter; hinter den unterhaltsamen Episoden dieses Filmes steht eine ernüchternde Wahrheit: Die Bewohner_innen zahlen einen viel zu hohen Preis, als sie Villa del Río ,zur Ware werden lassen“.

Als ziemlich à point servierte Kritik am sozial und wirtschaftlich zusammenbrechenden Regime Francos ist García Berlangas Versinnbildlichung heute aktueller denn je, wenn es darum geht, einem kaufkräftigen nordeuropäischen Mittelstand den Traum des ,typisch Spanischen' (Sonne, Strand und Fiesta) zu verkaufen: Das war das Entwicklungsmodell, das sich unter Franco etabliert hatte, um dem unaufhaltsamen Prozess des Industrieabbaus in Spanien entgegen zu wirken.[2] Heute gilt für Spanien: Die Geister, die ich rief, werde ich nicht mehr los.

Ein Spaziergang durch die heutige Stadt Málaga genügt, um Zeuge der Effekte der seit Jahrzehnten aggressiv praktizierten Tourismuspolitik zu werden. Die früher mit Autoverkehr und lokalen Geschäften belebte Avenida de la Constitución, wo Einheimische sich für ihre Alltagsbesorgungen mit Tourist_innen vermengten, ist zu einer Fußgängerzone gemacht worden, die von den überall vertretenen Modefranchisen gesäumt wird. Aus den Geschäften strömen Flyerverteiler, die im lauten Gedröhne der Technohits auf der Straße kaum zu verstehen sind. Gelegentlich sind Musikantengruppen zu sehen, die in Trachtenkostümen die in den Eiscafés sitzenden Tourist_innen mit folkloristischer Musik belästigen. Die die Straße flankierenden, für Málaga so charakteristischen Balkone sind nicht mehr zu erkennen, da sie mit zahllosen spanischen Fahnen verhängt sind. Weiter vorne erstreckt sich im Getümmel die ursprüngliche Altstadt, die längst einer architektonischen Aufwertungsmaßnahme zum Opfer gefallen und 
kaum noch bewohnt ist. Die unteren Etagen ihrer historischen Gebäude wurden in Kettenläden, Restaurants und Bars umgewandelt. Am anderen Ende der Avenida liegt der Sporthafen (puerto deportivo). Seit 1998 in einem tiefgreifenden Umbauprozess begriffen, umfasst dieser heute neben Anlegern für Fischerboote auch neue Stellplätze für Riesenkreuzfahrtschiffe und Yachten. Meeresschutt und tote Palmenzweige bedecken das Ufer der einst ruhigen Bucht von Málaga. Geplant sind genau hier ein großes Geschäftszentrum, ein Schiffsterminal der größten Kreuzfahrtschiffe der Welt der Firma Royal Caribbean sowie ein luxuriöses Wolkenkratzerhotel. Oben an der Autobahnzufahrt ist zur Zeit ein Plakat zu sehen, das für das Entwicklungskonzept der in der Kommune regierenden rechtskonservativen Parteien wirbt[3] und all diese Transformationen passend mit dem Slogan zusammenfasst: „Málaga, donde la cultura es capital“[4].

Das nicht ins Deutsche übertragbare Wortspiel mit ,capital', das sowohl ,Kapital' als auch ,Hauptstadt' bedeuten kann, wirft bezüglich der Diskussion darum, wie Städte zu ,Kapital' werden, einige Fragen auf. Zur Erläuterung braucht es zunächst eine konzeptionelle Präzisierung: Eine ,Stadt' ist nicht nur als zu füllender ,Container', als Ansammlung von Wohnbauten und Infrastrukturen anzusehen, so wie es früher in Spanien gedacht wurde, als die angeblich ,leeren' und ungenutzten Strände den Tourist_innen überlassen wurden. Mit dem Ansatz von Henri Lefebvre (1974) lässt sich die Stadt vielmehr als ein Lebenssystem verstehen, als ein materiell-immaterieller Komplex aus Beziehungsgeflechten, Repräsentationssystemen und Lebensweisen, welche an bestimmte Bau- und Nutzungsstrukturen gebunden sind, die den hier erwähnten kapitalakkumulativen Austauschprozess unterbinden. Die Doppelbedeutung von ,capital' veranlasst uns, die Vermarktungsstrategien, bei denen aus einer Stadt ein Mehrwert gewonnen werden soll, zu hinterfragen. Wie es auf dem Plakat steht, übernimmt diese Aufgabe im Fall Málagas schlicht die,Kultur. Zusammenfassend gesagt überschattet und überlagert der fortschreitende Kapitalisierungsprozess Málagas das Lebenssystem ,Stadt', indem er die ,Kultur primär als Marketingargument instrumentalisiert.

Es lohnt an dieser Stelle, sich auf die Vorlage dieser Gedanken zu besinnen. Schon Karl Marx erklärt in Das Kapital. Kritik der politischen Ökonomie (2003 [1867]), wie Gemeingegenstände zur Ware werden. Ähnlich wie bei der Stadt, die sich durch Kultur in Kapital verwandelt, wird bei Marx aus dem Gemeingut durch einen Hauch Rarität eine begehrenswerte Ware gemacht (vgl. ebd., Abschnitt „Warenfetischismus“). Eben dieser Raritätscharakter, der aus dem Anspruchsuniversum des Käufers entspringt, macht den Identifikationskomplex oder das Repräsentationssystem aus, das wir Kultur nennen (Appadurai 1986). Nicht von ungefähr sprach Marx vom ,Fetischcharakter der Ware', denn was konsumiert wird, ist nicht nur das Ding als solches, sondern auch das, was es darstellt. Wie die einfachen Dinge verwandeln sich auch Städte in Ware, wenn sie zur Tourismusdestination gemacht werden. Aus einer fiktiven Aura von Rarität und Attraktivität erzeugt, besitzen sie nicht nur einen Marktwert, sondern sollen auch die Erwartungen und Ansprüche des Käufers nach ,Kultur‘ erfüllen.

In Hinblick auf die Art und Weise, in der Málaga zurWare, zum Ort einer Erlebnisversprechung gemacht wird und wurde, bekommt diese These zentrale Bedeutung, denn sie erhellt die Prozesse, mit denen nicht nur Städte, sondern 
vor allem ihre Bewohner_innen und deren lebensstützende Strukturen als touristische Ziele, als Ware dieser Industrie, vermarktet werden. Tourist_innen erwarten belebende und inspirierende Orte, die sie in ihrem radikalen Anderssein aus ihrem Alltagstrott herausholen. Sie erhoffen sich ,exotische; fröhliche Momente, in denen sie am authentischen, bedeutungsvollen Leben eines Ortes teilnehmen dürfen (Zukin 2008), verbunden mit einer perfekt funktionierenden Infrastruktur. In Anbetracht des Ausmaßes, zu dem Málaga vom Tourismus geprägt ist, ist die obige Umdeutung des Begriffes ,Stadt vom Lebenssystem zur Ware hier von besonderer Relevanz. Das zeigt sich nicht zuletzt in einer Veränderung im Verhalten der Tourist_innen in ihrer Beziehung zur Stadt und ihrem Umgang mit ihr. Sie konsumieren längst nicht mehr nur deren Sehenswürdigkeiten wie Denkmäler oder Bauwerke, sondern die Stadt als Lebenssystem. Es ist genau diese Veränderung im Verhalten von Tourist_innen, von der wir annehmen, dass sie auf materieller Ebene für die Ausschöpfung und Enteignung[5] von Stadtressourcen und Logistik und auf immaterieller Ebene für das Verschwinden von Lebensweisen und Identitäten verantwortlich ist.

Aufwertung und Quartiersentwicklung haben also nicht mehr primär das Ziel einer Verbesserung der Lebensbedingungen der lokalen Bewohner_innen, sondern sollen genügend urbane Szenarios für Tourismuserlebnisse und Urlaubsdomizile bereitstellen. Vorbei sind die Jahre zwischen den 1960ern und 1990ern, in denen Tourist_innen nur mit dem üblichen Stranderlebnis zu unterhalten waren (Lefebvre/Regulier 2004 [1986]). Bei den Mittelmeerstädten ist ein Wandel im Trend der Tourismusziele zu beobachten (CocolaGant 2018: 2), der in Spanien nach der Logik des Werbeslogans Kultur ist Kapital einen beispiellosen Prozess der Stadtverdrängung ausgelöst hat. Angesichts der Lage in Málaga, wo in maroden und von Stadtpolitikern vernachlässigten Vierteln Menschen leben, die aus dieser Stadt politisch ausgeschlossen, jedoch zugleich (als Arbeitskräfte, Statist_innen, Bürger_innen) alternativlos an sie gebunden sind, wollen wir über Verhältnisse sprechen, die an Ausbeutung grenzen.

In Málaga kommen, ähnlich wie in vielen anderen spanischen Tourismusdestinationen (Russo/Scarnato 2018), zur Beeinträchtigung durch die vielen Besucher_innen die verhängnisvollen Effekte der Immobilienspekulation hinzu. Bezüglich der Entstehung von Leitkulturen und Ausschließungsmechanismen erkennen wir in den negativen Effekten, die der Massentourismus und die Immobilienspekulation auf Städte haben, Ähnlichkeiten zu kolonialen Verhältnissen. Im Gegensatz zu einigen Ansätzen der kritischen Tourismus- und Raumforschung (Coccola-Gant 2018; Blázquez-Salom 2013; Schäfer 2013; Smith 1990), die nur punktuell von zeitlich begrenzten kolonialen Verhältnissen zwischen Ursprungs- und Zielländern berichten, wollen wir - aufgrund der besonderen räumlichen und Nutzungsdimension der Problematik - auf Formen eines intraterritorialen Kolonialismus hinweisen. Was hier zur Diskussion steht, sind Phänomene einer Raum- und Bestimmungsenteignung, welche sich zwischen geopolitisch gleichwertigen Einheitenwie etwa der EU oder sogar intraurbanen Gebieten ereignen. Die Zentralität der Stadt als Produktionsraum für die Tourismusindustrie passt in ein Zeitalter, in dem Deindustrialisierung und Globalisierung als Grundlage neuer Prozesse der Ausbeutung fungieren. 
Wir gehen vor diesem Hintergrund davon aus, dass die wirtschaftlichen Praktiken von Aufwertung und Finanzialisierung von Stadtboden, die sich durch Privatisierung und temporäre Nutzung entwickeln und sich im Tourismus mit Strategien der Entwicklung einer gefälligen Leitkultur verbinden, Züge eines kolonialen Regimes aufweisen. Beim historischen Kolonialismus waren es die Diskurse der Modernisierung und des Fortschrittes, die die Praktiken des Outsourcings und der menschlichen Entfremdung ermöglicht haben (Henrique Martins 2018; Mignolo 2003; González Casanova 1965). Demgegenüber stehen hier Modelle für Stadtökonomien, welche die Bürger_innen an die Peripherie der eigenen Stadtbürgerschaft und Selbstbestimmung drängen. Dies ist besonders dort der Fall, wo europäische Ökonomien darauf setzen, mit Dienstleistungen und Erlebnissen zu handeln; gemäß der Idee, die Vermarktung von Städten durch touristische Produkte und kulturelle Events in den Mittelpunkt zu stellen. Innerhalb dieser Konstellation stellt die Stadt das Rohmaterial, die Ressource für diese Endprodukte dar. Wenn die negativen Effekte dieser Prozesse ein emanzipatorisches Potenzial bergen, lassen sie sich innerhalb des erwähnten Paradigmas eines intraterritorialen Kolonialismus betrachten. Es ist daher nicht verwunderlich, dass eine progressive Stadtpolitik in Spanien (wie die von der Bürgermeisterin Barcelonas Ada Colau, im Amt seit 2015) nicht ohne Bezugnahme auf die folgenden Faktoren auskommen kann: das Recht auf die Stadt und die Regulierung von Tourismusströmen und Immobilienspekulation.

Im Folgenden wollen wir das wirtschaftliche Segment des Tourismus und der Immobilienspekulation in Spanien unter die Lupe nehmen. Wir fragen, inwieweit die neuen Formen von Konsum und Vermarktung urbaner Zentren als „Themenparks in Lebensgröße“(Judd/Fainstein 1999: 89) einerseits die Stadt als Lebenssystem ausschöpfen und sie andererseits in ihrer demokratischen Legitimation entpolitisieren und entmächtigen. Wir sehen darin eine Besonderheit des spanischen Falls und begründen unsere These der Entstehung eines intraeuropäischen und intrastädtischen Kolonialismus wie folgt: Die negativen Effekte des Tourismus als Form von intraeuropäischem und intrastädtischem Kolonialismus ergeben sich aus seiner Verflechtung mit den Liberalisierungsmöglichkeiten, die der Immobilienboom von 1998 bis 2008 in Spanien für den Stadtraum und den Besitz über Boden mit sich gebracht hat.[6]

Im ersten Teil betrachten wir daher die Zusammenhänge zwischen der sogenannten Spekulationsblase und dem Aufkommen der city brands mit ihrer urbanen Umstrukturierung einerseits und den Entwicklungen im Tourismus andererseits; im zweiten Teil thematisieren wir potentielle Widerstandsbewegungen gegen diese Enteignungsprozesse am Beispiel von Málaga.

\section{Ausverkauf der Städte: vom Immobilien- zum Tourismusboom}

In Spanien entstand die touristische Kolonisierung nicht von einem Tag auf den anderen. Es handelt sich vielmehr um einen langsamen und sehr komplexen Prozess, dessen gegenwärtige Dominanz laut Ivan Murray (2015) in 
der wichtigen Funktion begründet liegt, die das spanische Staatsgebiet (der Raum) seit Mitte des vergangenen Jahrhunderts für das europäische und globale touristische Kapital erfüllt hat. Dies hat sich auch durch die Einführung der Demokratie in Spanien und dessen Eingliederung in die Europäische Union nicht grundlegend geändert. Die Akkumulationslogik des Tourismus spielte in der Diktatur gleichzeitig mehrere Rollen: „eine wirtschaftliche, als eine Art Marshallplan, nur mit Sonnenschirm und Sonnencreme; eine geopolitische, indem er als antikommunistische Schutzbarriere wirkte; eine propagandistische, indem er das Regime im Ausland rechtfertigte; eine soziopolitische, indem er durch die Verbreitung von Konsumkultur als soziales Schlafmittel wirkt“ (Murray 2015: 4). Die Zahl der Besucher_innen verzehnfachte sich innerhalb relativ kurzer Zeit (in etwa dem Zeitraum zwischen 1956 und 1960), wofür das Gebiet vorbereitet werden musste. Die Machtstrukturen des spanischen Tourismusunternehmertums begannen sich in Verbindung mit dem Franco-Regime zu bilden, gefördert durch internationales Kapital und lokale Machthaber sowie Spekulanten, und das in einer Zeit frenetischer Urbanisierung. Mit dem Wandel zur Demokratie ab 1975, dem Aufkommen des neoliberalen Projekts und dem EU-Beitritt 1981 (damals noch EG) verfestigte sich die Rolle Spaniens in der westeuropäischen Arbeitsteilung als Produktionsraum für Tourismus und Immobilien. Für die folgenden dreißig Jahre bis zur Finanzkrise schritt der Industrieabbau voran und im Kontext weltweiter finanzieller Liberalisierung etablierte sich eine Kultur des Finanz- und Immobilienbooms. Diese wurde ermöglicht durch das Gesetz zur Liberalisierung des Bodens von 1996, das den gesamten Boden, der nicht geschützt oder anderweitig festgelegt war, für bebaubar erklärte. In kurzer Zeit kam es daraufhin zum Wandel - der ,Tsunami ' der Urbanisierung war nunmehr unaufhaltsam. Das jährliche Volumen an fertiggestellter Geschossfläche stieg zwischen 1990 und 2005, also innerhalb von 15 Jahren, von 6,7 Millionen auf 10,2 Millionen Quadratmeter (Murray 2015: 7). Wie der langjährige Beobachter der spanischen Immobilienblase, Christopher Marcinkoski, bemerkt, übertraf die Neubautätigkeit in Spanien zwischen 1996 und 2006 die von Deutschland, Frankreich und Großbritannien zusammen (Marcinkosky 2015: 73).

Angesichts des Ausmaßes dieser fiesta wollte niemand auf der Strecke bleiben: Städte und Gemeinden konkurrierten darum, die meisten Megaprojekte, Wohnsiedlungen und Tourismuskomplexe zu realisieren, wofür sie auf alle möglichen Strategien zurückgriffen. Dabei wurde ein Korruptionsnetz zwischen Politik und Wirtschaft gewoben, dessen Ausmaß wir heute erst stückweise rekonstruieren können. Die Kooperation zwischen den Banken mit immer neuen, ad hoc generierten Finanzprodukten und der Politik, die die Gesetze an die ökonomischen Bedürfnisse der Investor_innen anpasste, wird nun deutlicher. Einerseits sahen sich die Gemeinden angesichts der fehlenden Kommunalfinanzierung dazu gezwungen, die Immobilienentwicklung als Hauptquelle steuerlicher Einnahmen zu fördern. Andererseits wurden neben den Flexibilisierungsmaßnahmen bei der verbesserten Möglichkeit der Bodenausnutzung (etwa durch veränderte baurechtliche Festsetzungen) Politikmaßnahmen verabschiedet, die den Investor_innen einen leichten Zugang zu Finanzierungskapital versprachen. Spanien hatte sein maßgeschneidertes Entwicklungskonzept endlich gefunden: Durch 
die Bewerbung und das Branding von Städten wurden Immobilienaktivitäten wie der Neubau intensiviert und die Tourismusindustrie über das klassische ,Strandprogramm“ hinaus gefördert. Zunächst als zweigleisige Idee eines Strukturwandels konzipiert, begannen die Kapazitäten der Bauindustrie mit den Zielen der Tourismusbranche zu konvergieren.

Die Kommodifizierungsfolgen für die Städte waren enorm: Sie wurden für die städtischen Imagestrategien immer mehr zur Marke. Diese Strategien hatten vor allem das Ziel, die Attraktivität der Städte in Hinblick auf die Immobilienentwicklung zu erhöhen. Zeitgleich zum bereits erwähnten Zenit der Immobilienspekulation wurde 2008 ein historisches Hoch von spanienweit 58,6 Millionen Tourist_innen erreicht. Neben der plakativen Darstellung der Beliebtheit bestimmter Städte als Tourismusziel zählen zu den Gründen für diese Entwicklung zum Beispiel auch die Zunahme von Billigflügen und der Bau neuer Flughäfen, die nach der Finanzkrise sogar zum Verkauf standen. Laut der Tageszeitung El Mundo kommerzialisierte beispielsweise der spanische Flughafenbetreiber AENA, heute eine Aktiengesellschaft, im Jahr 2017 mehr als 2.0oo Hektar für den Bau von Hotels (Segovia 2017).

Durch das Einsetzen der Weltwirtschaftskrise, in der die Finanz- beziehungsweise Immobilienblase platzte, wurde das soziale Gewebe der spanischen Gesellschaft angegriffen; die Krise hinterließ verwüstete Landstriche in ganz Spanien: Geistersiedlungen, halbfertige, verfallene Gebäude, Kreisverkehre mitten im Nirgendwo. Ende 2010 standen über eine Million Neubauten leer. Und es hätten sogar 3,6 Millionen werden können, wären alle genehmigten Bauprojekte tatsächlich umgesetzt worden (Marcinkoski 2015: 73). In Anbetracht der Tatsache, dass die Wohnsiedlungen für die durch die Krise verarmten lokalen Käufer_innen unerschwinglich geworden waren, richtete die Bauindustrie nun ihr Augenmerk auf die Schaffung von Räumen für den Tourismus und damit auf die erzwungene Ästhetisierung des Raumes zu kommerziellen Zwecken. In dieser Hinsicht findet in Spanien derzeit eine Art paradoxer Vorgang der urbanen An- und Enteignung statt - im Schoß eines ökonomischen Modells, in dem die Profite und Privateigentum schaffenden Mittel von Tourismus- und Immobiliengeschäft zusammenwirken.

\section{Tourismusstädte: Wo Kultur ist, ist Kapital}

In Málaga hat ein neues Modell von Stadt den städtischen Raum buchstäblich erobert. Es basiert auf Konsum und der Schaffung von touristischen Räumen. Um dieses Modell zu etablieren, hat die Stadtverwaltung in den vergangenen zehn Jahren eine Reihe von Maßnahmen initiiert, die Málaga auf dem Markt der internationalen Touristenhochburgen platzieren sollen, indem sie sich oberflächlicher Mechanismen bedienen, die nur auf das Spektakel abzielen. Die Entstehung des Entorno Thyssen in Málaga, die Erweiterung des Hafens 2012 für das Anlegen von Kreuzfahrtschiffen und Yachten, die Zunahme von Genehmigungen für Bars, von Kettenläden und Ähnlichem im Stadtkern, der Bau von Museums-Franchiseprojekten, denen die Verbindung zur lokalen Kultur fehlt, wie dem Centre Pompidou Málaga 2015, und die Etablierung von sogenannten alternativen Touren durch die ärmeren Stadtviertel zur Besichtigung der von gewöhnlichen Graffitis geprägten Stadtlandschaften sind nur einige Beispiele hierfür. 
Stadt und Kultur werden dabei als Marktinstrumente verstanden. Nichts bezeugt dies stärker als die Werbeslogans auf Tourismusmessen: „Málaga, wo Kultur Kapital ist“, oder die aktuelle Version: „Málaga, Stadt der Museen“. In Wirklichkeit handelt es sich jedoch um eine Stadt als Museum (eine Unterart der Stadt als Marke), also eine Stadt, die den Besucher_innen als Ware zurVerfügung steht und in der das, was sich hinter der Fassade befindet, keine Rolle mehr spielt. In der Stadt als Museum gibt es kaum noch Bürger_innen (und wenn, dann nur in folkloristischer Tracht oder als Servicepersonal), sondern fast nur noch Tourist_innen, Geschenkläden und Restaurantketten. Die Stadt und die Bürgerschaft verschwinden, es bleibt nur die Illusion, ein Trugbild der Stadt. Diese Entwicklung vertuscht die Vertreibung der Mehrheit ihrer Einwohner_innen durch Enteignung und Veräußerung. Was die Strategien betrifft, nimmt die Stadtverwaltung keine passive Haltung ein, sondern ganz im Gegenteil: Sie spielt eine aktive Rolle, denn sie verwaltet die Enteignung.

In Málaga nimmt in diesem Zusammenhang die Privatisierung von Stadtraum seitens der Tourismusinvestoren $\mathrm{zu}$ - proportional zur Enteignung der Stadtbewohner_innen. Diese suchen sich, entweder aufgrund der Mietpreise oder wegen der Undurchführbarkeit eines Lebens in räumlicher und Nutzungskonkurrenz zu den Besucher_innen, anderswo ein $\mathrm{Zu-}$ hause. Dies ist zunächst eine Konsequenz der staatlichen Förderung von Immobilienerwerb, aber ganz entscheidend auch ein Effekt der tatkräftigen Enteignung von urbanem Raum mit dem Ziel, touristische und freizeitwirtschaftliche Erlebnisräume zu erschaffen. Zum einen hat die Tourismusindustrie Städte als Lebenssysteme durch eine markenhafte Nachbildung ihrer selbst zu Kulissen werden lassen. Die komplexe und mannigfaltigere Materialisierung des Lebens, das letztendlich eine Stadt ausmacht, wird durch die Zweidimensionalität eines Logos ersetzt; das sensiblere Gleichgewicht wird durch ein einziges Zielprogramm und eine passende Leitkultur gefährdet. Zum anderen nutzt die Immobilienbranche die An- und Enteignungsmöglichkeiten eines optimierten Immobiliengeschäftsmodells, das aufgrund des Bodengesetzes von 1996 und der zunehmenden Deregulierung bei der Umwidmung von öffentlichen Flächen und Gemeinbedarfseinrichtungen die Einrichtung von Ferienwohnungen im Mietwohnungsbestand der Städte möglich gemacht hat.

Die Idee, aus der Stadt eine Marke zu machen, ist tief ins Netz der Straßenführungen vieler spanischer Städte eingesickert. Dies zeigt sich ganz deutlich an Phänomenen wie der curated streets oder der ,Touristenrouten', die in Städten wie Barcelona, Sevilla und Málaga ganze Straßen und Quartiere umund erschließen. In Málaga, im sogenannten Entorno Thyssen, verfügt zudem Carmen Cervera, die Witwe und Erbin der Kunstsammlung des Grafen Thyssen-Bornemisza, über die Bestimmungs- und Entwicklungsrechte von kommerziellen Lokalen in der Umgebung des Gebäudes, in dem die Sammlung gezeigt wird. In diesem Stadtteil befindet sich der im Stadtvergleich höchste Anteil an Wohneinheiten, die als temporäre Unterkünfte gekauft wurden und entsprechend ihrem Spekulationswert nicht als permanente Wohnungen genutzt werden. Dabei wird ein Stadttypus geschaffen, der, von Tourist_innen genutzt, sich immer,im Durchlauf' befindet. In Málagas historischem Stadtkern zum Beispiel übertrifft heute die Anzahl der temporären Privatunterkünfte wie die der Plattform Airbnb jene der 
Wohneinheiten, die für die ortsansässige Bevölkerung bestimmt sind: Von seinen ca. 1.319 Gebäuden[7], unter denen auch zahlreiche Ladenlokale, Geschäfte, Hotels, Museen, Restaurants usw. sind, wurden mehr als 300 von 450 Wohneinheiten ausschließlich Airbnb überlassen. Der explosionsartige Touristenboom schlägt sich, durch die Vermietung von Wohnungen für den Tourismus, in einem Anstieg der Mieten nieder. Diese Zweckentfremdung von Wohnraum durch Plattformen wie Airbnb hat beispielsweise den VFTVerband (viviendas con fines turísticos oder Unterkünfte für touristische Zwecke) hervorgebracht, der sich zum Großteil auf das Angebot von Unternehmen mit großen Wohnungsbeständen konzentriert.[8]

Auch die Besiedlung des öffentlichen Raums durch Bars und Restaurants im Stadtzentrum wird hauptsächlich mit MAHOS (Verband der Hoteliers von Málaga) in Verbindung gebracht. Dieser mächtige Verband setzt sich unter anderem gegen eine Regulierung von Lärm ein, also dagegen, das Zentrum zur ,akustisch gesättigten Zone` zu erklären, und seine Mitglieder verstoßen systematisch gegen die geltenden Vorschriften in Bezug auf die Ausdehnungen ihrer Terrassenbereiche.[9]

Auf die Qualität der Berufsperspektiven der Bürger_innen haben diese Entwicklungen ebenfalls gravierende Auswirkungen: Das Angebot an Arbeitsplätzen, das das Tourismusgeschäft mit sich bringt, schafft Beschäftigungsarten, die zu den prekärsten zählen. Zudem führt es zu einem zunehmend einseitigen Arbeitsmarkt, auf dem Innovation und Umweltbewusstsein fast nie gefragt sind. Die Rede ist hier von Saisonarbeiter_innen, Kellner_ innen, Animateur_innen, Reinigungspersonal und so weiter, die oft nur im Sommer und unter Arbeitsbedingungen arbeiten, die mit ihren niedrigen Löhne und dem Schichtdienst an Sklaverei grenzen. Wie häufig an der Küste der Fall, kommen diese Saisonarbeitskräfte entweder aus anderen, wirtschaftlich schlechter gestellten Provinzen Spaniens oder aus anderen Stadtgebieten in einen Stadtraum, den sie nach Arbeitsende verlassen müssen. Für sie ist die touristische Stadt eine Phantasie, die sie nähren, an der sie jedoch nicht teilhaben. Laut einer Studie von EXCELTUR (alianza para la excelencia turística, ein Verein zur Förderung und Erforschung des Tourismusgeschäfts in Spanien) aus dem Jahr 2015 machte der Anteil an Arbeitsplätzen in der Tourismusindustrie beim so genanntem nicht qualifizierten Personal in Spanien 89,8 Prozent der insgesamt geschaffenen Arbeitsplätze aus. Übertroffen wird diese Zahl nur durch den im Landwirtschaftssektor (Exceltur 2008). Auf den Alltag im Quartier haben diese neuen Formen von stadtumgreifendem Tourismus fatale Auswirkungen: So ist etwa die Förderung von immer mehr Straßenfesten, Festivals usw. Teil dieser undemokratisch gesteuerten und den Alltag beeinträchtigenden Vermarktungsstrategie. Zur Steigerung ihrer touristischen Attraktivität erfinden religiöse Institutionen in manchen Städten Andalusiens und der Levante sogar neue folkloristische oder religiöse Veranstaltungen. Religiöse Prozessionen und Züge vermehren sich unerklärlich, in Form von längst vergessenen oder ad-hoc erfundenen Traditionen wie la batalla de las flores (eine Art Blumenkampf, bei dem die Menschen sich mit Blumen bewerfen) oder el via crucis de Agosto. Dafür sperrt die Stadt die Straßen für den normalen Verkehr, außerdem beanspruchen die Prozessionen eine beträchtliche Menge an Notfall- und Sicherheitspersonal. Dies gilt auch für die offene 
Förderung von bestimmten Arten von Wettbewerben und Ritualen wie dem sogenannten balconing [10] (bei dem es darum geht, vom Balkon aus in einen Swimming Pool zu springen) oder der Junggesellenabschiede, bei denen exzessiv und unter missbräuchlicher Nutzung des öffentlichen Raumes gefeiert wird.

Wie wir anhand der obigen Beispiele zu erläutern versucht haben, ergibt sich durch die neuen, auf Tourismus und Konsum ausgerichteten Nutzungen der Stadt eine Situation von Nutzungskonkurrenz im Stadtraum. Diese basiert auf Identität und Subjektivierungsvorherrschaft. Die Bürger_innen verlieren nicht nur die Rechte zur Nutzung und Gestaltung ihrer Umgebung, sondern ringen mit den Tourist_innen um dieVorherrschaft über die geteilten Räume, wie die in Barcelona ansässige Plattform El Turisme Mata Els Barris anprangert. Man bedenke, dass Barcelona mit einer Einwohnerzahl von 1,6 Millionen mit jährlich 8,36 Millionen Tourist_innen (und allein im Juli 773.958 Besucher_innen) ein besonders krasses Verhältnis von Tourist_innen pro Einwohner_in ausweist (Statista 2018). Die touristischen Aktivitäten werden meist von privaten Akteuren angeboten. Auch wenn der Tourismus eine wichtige Einnahmequelle für spanische Städte ist, profitieren die Einwohner_innen davon nur marginal. In der traditionell neoliberal regierten Stadt Málaga ist die Lage besonders gravierend. Die Bürger_innen zahlen zwar die Kosten für verbrauchte Ressourcen, die für Wohnflächen und öffentliche Flächen benötigt werden, aber haben selbst wenig von den staatlichen Investitionen.

Durch das Versprechen eines exotischen Erlebnisses, mit dem Besucher_innen heute angelockt werden, wird die Touristenstadt, wie oben erwähnt, zum Inbegriff des intrakolonialen Territoriums, in dem Prozesse des Machtgewinns und des ästhetischen Raumkonsums mit solchen der Segregation und der Ausgrenzung einhergehen.

\section{City brands und die Enteignung von Stadtraum}

Der Stadttheoretiker Wolfgang Scheppe, der sich mit einer ähnlichen Problematik im Fall Venedig beschäftigt, fasst diese Wende adäquat so zusammen, dass „der urlaubende Fremde als primäre Einkommensquelle und die Stadt mit der diffusen Verheißung urbaner Außergewöhnlichkeit als in ihrer Totalität zu vermarktendes Konsumgut gilt“" (Scheppe 2018: 22). Die Stadt, so Scheppe weiter,

„ist zu einem Markt Internet-vermittelter Kurzzeitbehausungen geworden. Straßen, Läden und Infrastrukturen erweisen sich dort zunehmend als nunmehr dem transitorischen Betrachter zugewandte Kulisse eines ihm zugemessenen Warenangebots. Demnach ist die Stadt als Finanzinstrument nicht mehr als eine Metapher ihrer Selbst [sic].“(ebd.: 27)

Die Auswirkungen dieser Art von Enteignung verdienen besondere Aufmerksamkeit, denn sie bedeuten eine faktische Machtausübung seitens der Stadteliten und Immobiliengesellschaften, die aber selten juristisch dargestellt wird: Die Stadtbewohner_innen bleiben in den Städten ,eingeschlossen', ohne ein politisches Recht auf diese zu haben. Mit Hilfe von Steuervorteilen erzielen die Agenten der Privatisierung ihre spekulative 
Rendite aus dem Erwerb und Verkauf der enteigneten Immobilien, die von Hand zu Hand gehen. Dies spricht eher für eine Profitmaximierung durch die relativ konkrete Umnutzung und Umwandlung der Gebäude. Ein verbreiteter Vorwurf ist dabei, dass sie, obwohl sie politisch die Oberhand über die Stadt und ihr ökonomisches Schicksal haben, keine materielle persönliche Verbindung mit ihr anstreben oder besitzen. Unsere Argumentation zielt jedoch darauf ab, Formen der ,immateriellen Enteignung', die mit diesen Prozessen einhergehen, herauszuarbeiten.

Durch Immobilienspekulation, den exponentiellen Anstieg von Mietpreisen und das Verschwinden von lokalem Gewerbe und Dienstleistungen für die alltägliche Versorgung werden die Einwohner_innen aus ihren Wohnorten bzw. dem öffentlichen Raum gedrängt, entwurzelt und ihres Rechts auf die Stadt beraubt. Gleichzeitig sehen sie sich unwiderruflich eingeschlossen und gefangen in eben diesem nun verwandelten Raum, den sie, zumindest als Passant_innen oder Statist_innen, nicht umgehen können. Gerade in dieser Ambiguität von Enteignung und Ausschließung einerseits sowie Ausgrenzung, Vertreibung und Einschließung andererseits realisiert sich eine Art von Herrschaft, in der der urbane Raum zur eigenen Umgrenzung, zur eigenen Umzäunung und somit zum primären exekutiven Apparat dieser Herrschaft wird. In der letztlich ,entpolitisierten Polis', einer Umzäunung in Lebensgröße, werden die Einwohner_innen gleichzeitig aus den Stadtzentren ausgegrenzt und in sie eingeschlossen; sie sind nunmehr in einem Raum gefangen, auf dessen Nutzung sie kein Anrecht haben, an dessen neuen Nutzungsarten - einem rein ästhetischen und trugbildlichen Konsum - sie jedoch gezwungenermaßen teilnehmen.

Wir stehen hier vor einer eigentümlichen Konstellation von politischen und wirtschaftlichen Elementen, in der Städte und ihre Einwohner_innen in hübsche, käufliche Postkarten, in Marken oder Embleme umgewandelt werden, die in der Wandlung vom sogenannten Liberalismus zum Neoliberalismus eine Parallele findet; ein System, das darauf basiert, mit Immateriellem zu handeln, also mit Bildern, Blasen, Spekulationswerten, Schulden usw., und bei dem die alte fordistische Trennung zwischen Realwirtschaft (Verkauf von materiellen und immateriellen Produkten) und dem monetären Finanzsektor (spekulative Strömung, gesteuert durch Entscheidungen von Investoren) aufgehoben ist und nur durch die Generierung von optimistischen Bildern im Scheingleichgewicht, immer am Rande des Kollapses, aufrechterhalten wird (Marazzi 2008). Bei dieser Umwandlung der Stadt in eine immaterielle Ware wird in der Logik des Finanzkapitalismus, wie Marazzi ihn nannte, die Entmaterialisierung, von der Marx sprach, wie folgt artikuliert: Tatsächlich wird privates Eigentum geschaffen, dieses fußt jedoch auf der ungreifbaren Transformation der Bürger_innen in Charaktere, pittoreske Gefangene von Städten, die sie materiell nicht mehr besitzen.

Schon David Harvey warnte vor dem sonderbaren Charakter des Bodens als Ware. Er ist, so sagt er in Die urbanen Wurzeln der Finanzkrise (2012),

„eine fiktive Form des Kapitals, die sich aus den Erwartungen auf zukünftige Miet- und Pachteinnahmen ableitet - die Rendite leitet sich demnach vom Möglichen ab, vom An- und Verkauf von Risiko und Verschuldung, und nicht etwa vom Besitzerwechsel der Grundstücke.“ (ebd.: 2) 
Hierauf baut die Maschinerie der Darstellung von Identitäten und gefälliger Symbolik auf, die einen belanglosen Ort in eine attraktive touristische Destination - eine city brand - verwandelt.

Es handelt sich bei der Stadt nun um eine Art von Privatbesitz, um deren materiellen Erhalt man sich nicht kümmert, sondern deren Wert man sozusagen auflöst, indem man ihr Potenzial für Subjektivierungen, Eigenbesitz und Bewegungsfreiheit einschränkt oder sanktioniert. Das Ziel ist, dass die Stadt sich in ein ansprechendes Bild für Tourist_innen verwandelt. Es handelt sich um ein Besitzverhältnis, das in fataler Weise an jenes zwischen Eigentümer_innen und Enteigneten in der Kolonialzeit erinnert: eine Herrschaft nicht nur über Territorien und die darin befindlichen Körper, sondern auch über Identitätsbildung und Mobilität, die sich intraterritorial, also zwischen verschiedenen Gruppen eines Territorium, etabliert.[11]

Daher ist es nicht verwunderlich, dass sich, wie Sharon Zukin (2014) darlegt, ein Zusammenhang zwischen dem Phänomen der city brands und der Entwicklung und dem kommerziellen Prestige der Weltausstellungen, die aus dem Rausch des Kolonialstolzes Mitte des 19. Jahrhunderts hervorgingen, erkennen lässt (Zukin 2014). Verbunden mit dem Versprechen der Stadterneuerung und der Präsentation architektonischer Entwürfe, die leicht mit Fortschritt assoziierbar sind, liegt der Schatten des Kolonialismus und der Segregation auf verhängnisvolle Weise über dem Phänomen der city brands.

Barbara Kirshenblatt-Gimblett (1998), die die Erforschung von touristischem Verhalten als Feld für die ethnologische Untersuchung von Machtverhältnissen bereits Mitte der 1990er Jahre vorschlug, hinterfragt in ihrem Werk die bislang wenig umstrittene Praktik, Kulturen zu musealisieren. Im Kapitel „Exhibiting Jews“ (ebd.: 79 ff.) schreibt sie, es genüge nicht, Artefakte zu präsentieren, um ein glaubwürdiges Bild einer Kultur zu vermitteln. Stattdessen müssten sowohl Gegenstände als auch Lebensformen kontextualisiert werden. In einer Kontextualisierung, Reproduktion bzw. Rekonstruktion, die auf eine von der Zeit entkoppelte, vom Wirklichen entrückte Kontemplation abzielt, würden nicht nur die Artefakte, sondern auch die Menschen musealisiert. Daher, so Kirshenblatt-Gimblett, hätten viele Museen Mumien oder lebensechte Puppen in ihre Inszenierungen eingebaut und ganze Siedlungen mit Dioramen ihrer Bevölkerung rekonstruiert, die die Szene beleben und ihr Sinn geben sollen. Die ausgestellten Jüdinnen und Juden bedienen in ihrem Beispiel einen anachronistischen Voyeurismus - nicht wie bei ihrem ersten Auftritt als Tragödie, sondern nun als Farce.

Wenn sich eine kolonialistische Komponente in dieser Praxis der Ausstellung von Kulturen innerhalb der Grenzen des Museums feststellen lässt, dann umso mehr, wenn diese auf die reale Stadt übergreift und dabei die Stadt in einen Ausstellungsort ihrer selbst verwandelt, wo ihre Lebensform eine totale Musealisierung erfährt, eine totale Reduzierung auf den touristischen Konsum.

Das Konzept der city brands, das vom Schaufenstereffekt der Weltausstellungen inspiriert ist, beruht auf der Idee, alles Menschliche lasse sich in einer Situation enger Identitätsumgrenzung ausstellen, aus der sich schließlich - so die Gefahr - eine territoriale Segregation entwickeln kann.

Der Fall der Weltausstellung von Brüssel 1958 zeigt, welch extreme Formen dieses Phänomen annehmen kann (Blanchard/Boëtsch 2012). Neben 
avantgardistischer Architektur wie dem Philips-Pavillon von Xenakis, Varèse und Le Corbusier, in dem die ersten Beispiele elektronischer Musik zu hören waren, stellte dort die eigentliche Touristenattraktion ein Menschenzoo dar, der eigens für den feierlichen Anlass errichtet wurde und das Leben in einem Dorf in Belgisch-Kongo inszenierte. Die Presse geizte nicht mit Lobeshymnen auf die Nachbildung, für die nicht nur die Hütten, sondern auch die leibhaftigen Einwohner_innen in anachronistischer Stammestracht in die mitteleuropäische Hauptstadt versetzt wurden. Ein Foto, das einen der euphorischen Berichte illustriert, zeigt, wie ein circa sechsjähriges kongolesisches Mädchen eine Banane annimmt, die ihr eine Frau über den Zaun hinweg reicht, der den Menschenzoo umgibt. Im Hintergrund applaudieren Besucher_innen begeistert und werfen dem Kind weitere Bananen zu, verbunden mit dem Wunsch, ebenfalls an dieser zärtlichen Szene der Begegnung und Dankbarkeit teilzunehmen, der rührenden Umarmung zwischen Zivilisation und Barbarei.

\section{Wiederaneignung: Rebellion im Zoo}

Seit 2011, dem Ende der Protestcamps der Bewegung Movimiento 15-M in Spanien, als die Bürger_innen in Folge der Krise und aus Unzufriedenheit mit der Regierung zunehmend auf die Straße gingen, hat sich eine Explosion von außerparlamentarischen, sogenannten Gegenmachtsinitiativen entwickelt. Diese treten in Form dauerhafter Hausbesetzungen und vorübergehender Besetzungen des öffentlichen Raumes in Erscheinung sowie durch die Bildung von Anwohnerversammlungen (asambleas) in den Stadtvierteln. Auf die Vorstöße der Immobilienmächte durch die touristische Kolonisierung reagieren die Bürger_innen mit neuen Praktiken der Wiederaneignung von urbanem Allgemeingut wie leeren Grundstücken, verlassenen Gebäuden und Brachen. Mit der Zeit haben sich einige Initiativen zerstreut, andere sind weiterhin aktiv und entwickeln neue mikropolitische Praktiken der Koordination, mit denen sie die Wiederaneignung auf eine neue Ebene bringen. Ihre Aktionen dienen dazu, die Enteignungsprozesse in der neoliberalen Stadt zu reflektieren und ihnen entgegenzuwirken. Die genauen Zusammenhänge und Verfahren dieser Praktiken der Enteignung zu verstehen, erscheint uns als notwendige Grundlage für jeden Versuch, Alternativen zu entwerfen.

Parallel zum Umbau der Stadt wurden an verschiedenen Orten in Málaga und zu verschiedenen Zeitpunkten politische Aktionen gestartet, durch die der vom Tourismus vereinnahmte urbane Raum zurückerobert werden soll. Wir sprechen von der oben erwähnten Art von Initiativen, die sich in der Stadt etabliert haben, beispielsweise in Gestalt von „La Casa Invisible“, dem unsichtbaren Haus, das im März 2007 mitten im historischen Stadtkern (Calle Nosquera 9-11) ins Leben gerufen wurde, als ein verlassenes Stadtpalais aus dem Jahre 1876 besetzt und in ein soziales und selbstverwaltetes kulturelles Zentrum unter Bürgerleitung umgewandelt wurde. Damit wollten die Anwohner_innen gegen die Enteignungsprozesse ankämpfen, die in Málaga damals bereits seit Jahrzehnten im Gange waren. Die Casa Invisible stellt ein eigentümliches Verdichtungsphänomen dar, bei dem alle drei in diesem Artikel behandelten Aspekte eines kolonialen Verhältnisses von Tourismus 
und Stadtbewohner_innen wirksam werden: die Verbindungen zwischen Immobilienboom und -krise, Tourismus und Widerstand. Von besonderer Brisanz ist, dass die Casa Invisible sich inmitten des Tourismusgewirrs im Zentrum von Málaga befindet. Seitens der Stadtregierung aus Partido Popular und Ciudadanos existieren seit 2017 Pläne für den Umbau des Gebäudes.

Auch andere Projekte wie der urbane Gemeinschaftsgemüsegarten „El Caminito“ sind im Zuge des 15-M und seinen Nachwirkungen entstanden. Ein ungenutztes, brachliegendes Grundstück im Stadtzentrum (CalleZurbarán 3) wurde dafür wiederbelebt und begrünt. Auch nach über zehn Jahren trägt „El Caminito“ heute noch Früchte. Die Aktionen der Anwohner_innen im Viertel Lagunillas und um die Calle Gigantes sind aber vielleicht das beste Beispiel für den Widerstand gegen die Tourismusindustrie, deren Ausschlüsse und deren Kolonisierung.

Das Viertel Lagunillas grenzt an den historischen Stadtkern und ist von den Plänen der Stadtverwaltung zur Ausweitung touristischer Ziele in der Stadt betroffen; entsprechende Pläne stehen auf EU-Ebene bereits zur Diskussion. Dies hat jedoch die Anwohner_innen nicht davon abgehalten, sich zusammenzuschließen, um ein leerstehendes Grundstück zurückzugewinnen, das bis dahin informell als Parkplatz genutzt worden war, und es in einen Raum für die Allgemeinheit zu verwandeln. In sämtlichen damit zusammenhängenden Demonstrationen sprachen sich die Bürger_innen 2016 kategorisch gegen die in den Plänen vorgesehene Bebauung aus und besetzten das Grundstück, das seitdem als Symbol und Raum für AntiGentrifizierungskampagnen fungiert. So entstand dort im Dezember 2016 das Gelände „Victoria, de quién?“ (doppeldeutig im Spanischen: „Wem gehört Victoria?“ und: „Wessen Sieg?“)[12], wo nun schon seit einem Jahr keine Autos mehr parken: Das Gebiet ist nun ein Ort für Vergnügung und Unterhaltung, aber auch politische Versammlungen. Es finden zum Beispiel Open-Air-Filmvorführungen und informelle Anwohner_innenversammlungen statt. Kürzlich kam es zu einer besonders geistreichen Aktion, als im Sommer 2017 ein Reiseveranstalter eine Tour durch das Viertel plante, bei der die Malereien und Graffitis der Gegend gezeigt werden sollten: Die Anwohner_innen verkleideten sich als Tourist_innen und veranstalteten eine Gegentour, in der das Objekt ihrer Besichtigung, das sie fotografierten und beobachteten, eben jene Tourist_innen waren, die das Viertel erkundeten. Auf diese Weise wurde das naive Vorhaben, ein , aufstrebendes Viertel ${ }^{\circ}$ mit seinem, Charme' zu besichtigen, unterlaufen: Die Tourist_innen, mit den eigenen Waffen geschlagen, wurden dazu angeregt, ihr scheinbar harmloses Handeln zu hinterfragen.

Anhand dieser Praktiken der Wiederaneignung sehen wir, wie die Mittel des Gegners zur Selbstverteidigung angewandt werden können: Innerhalb des Menschenzoos besteht die Möglichkeit, dass die Beobachteten und Musealisierten sich ihres Zustandes der Verdinglichung bewusst werden und reagieren, indem sie wiederum die Besucher_innen verdinglichen Rebellion im Zoo. Die Proteste finden in einfallsreichen Formen Ausdruck, um verdinglichend zu ,entdinglichen'; die Enteignung zu umgehen, indem man sich urbanes Gebiet wieder aneignet und so den Enteignungsversuchen von Allgemeingut durch eine, Recommunalisierung' (im Sinne von commons) entgegentritt. Den Anwohner_innen, die die Gegentour durchführten, welche 


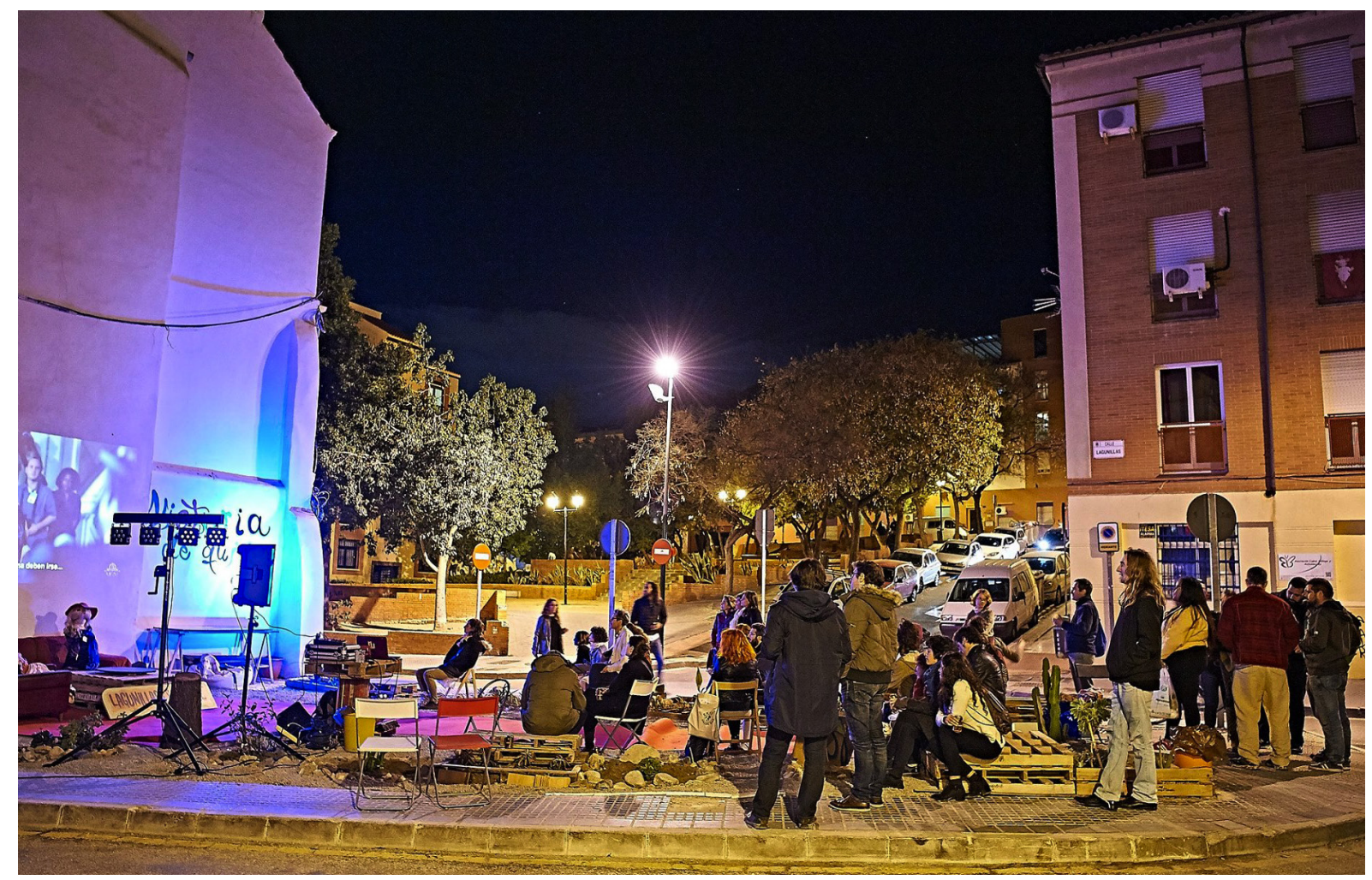

Abb. 1 Baulücke als Bauplatz „Victoria ¿de quién?" (Quelle: Juan Ortiz Rivas) zunächst ein Moment der Abruptheit in der Normalität der Besucher_innen darstellte, gelang es, eine neue Perspektive auf das, was im Viertel geschieht, zu eröffnen. Einige der Tourist_innen brachen sogar die Tour ab und kamen mit den Anwohner_innen ins Gespräch über die erlebten Probleme der Enteignung. Die anfängliche Schroffheit der Aktion wich einer sanfteren Beilegung, bei der die Besucher_innen aufhörten, Kompliz_innen der Enteignungsprozesse zu werden. Sie blieben zwar Besucher_innen, blickten aber etwas tiefer, hinter die glatte Fassade, die ihnen sonst nur geboten wird.

Das letzte und neueste Beispiel für die Praktiken der Wiederaneignung ist das Grundstück Gigantes, eine Fläche von 2.00o Quadratmetern, die jahrzehntelang vom Eigentümer_innen brachliegen gelassen worden war und schließlich durch eine Bürgerinitiative im Juni 2017 zurückgewonnen wurde. Die Anwohner_innen der Straße Calle Gigantes und deren Umgebung bewiesen ein enormes Organisationstalent, als sie im Rahmen einer Besetzung viele der Angebote und Aktivitäten, für die sie das Grundstück in Zukunft nutzen wollten, kurzerhand selbst auf die Beine stellten: Durch die Erschaffung von Sportplätzen über urbane Gemüsegärten bis hin zu kulturellen und pädagogischen Veranstaltungen gelang es ihnen, Netzwerke der Solidarität und der gegenseitigen Unterstützung zu weben, die das Ziel haben, Prozessen der Gentrifizierung entgegenzuwirken.

All diese Initiativen haben etwas gemeinsam: den Wunsch, die Stadt zurückzuerobern und sich das Gemeingut wieder anzueignen. In den vergangenen Monaten haben sie einander ihre Solidarität bekundet; sogar ein gemeinsamer Slogan wurde gefunden: „\#MálagaNoSeVende“ („Málaga steht nicht zum Verkauf"), der sich an ähnlichen Initiativen in Madrid und Barcelona und anderen (süd-)europäischen Städten orientiert (Rom, Venedig, Neapel, Lissabon, Athen etc.). Womöglich könnte die Bewegung zu einer Mobilisierung der Bewohner_innen der Metropolen avancieren, die ausgehend von Vielfalt und Autonomie den Ungehorsam koordinieren 


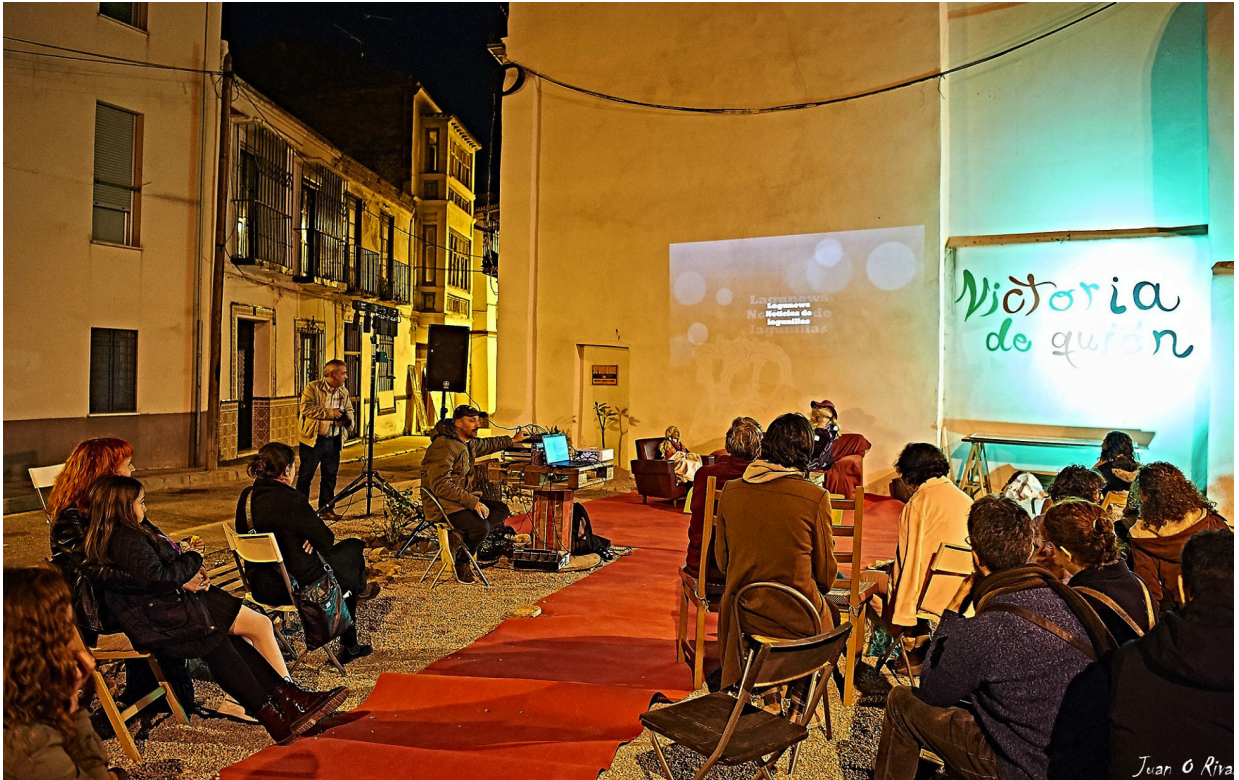

und zeigen könnte, dass in den Rissen der Touristenstadt noch eine andere Stadt existiert.

Diese Aktionen sind eine räumlich sichtbare Manifestation, doch gleichzeitig ist das, was hier am Werk ist, ein Prozess der Delegitimierung der heutigen Stadt und ihrer Logiken. Dieser Prozess definiert das soziale Imaginäre über das Recht auf die Stadt neu. Wie Amador Fernández-Savater, der Philosoph und in die 15-M-Bewegung involvierte Stadtaktivist, uns in einem Vortrag (2015) sagte:

„Jedes Mal, wenn einem Mieter die Miete erhöht wird, wenn eine Schule unter Kürzungen leidet, ein Krankenhaus von der Schließung bedroht ist, wenn ein Migrant keinen Zugang zum Gesundheitssystem hat, wenn eine Frau im öffentlichen Raum belästigt wird und einer Familie die Wohnung gekündigt wird, jedes Mal stellt sich dabei eine ganz praktische Frage:,Wie werden wir leben?‘ [Cómo vamos a vivir?]“

Die beschriebenen Aktionen stellen Wiederaneignungen dar, nicht nur des Raumes, sondern auch des Lebens. Sie realisieren sich mit Körpereinsatz, bestehen auf dem Recht auf Stadt und verlangen ein würdigeres, lebbareres Leben. Gleichzeitig entwerfen diese versammelten Körper eine neue Art zu leben, die niemanden außen vor oder im Stich lässt. Das bedeutet Kritik, Widerstand und Einfallsreichtum auf einen Schlag. In jeder einzelnen dieser Gesten steckt zudem eine stille und unvorhergesehene Freude, die die politische Grammatik und die Grenzen des Möglichen überschreitet. Eine Freude, die aus der Begegnung entspringt; daraus, gemeinsam aus dem tiefen Schlaf des Neoliberalismus aufzuwachen. Was sich dann zeigt, ist das kollektive Bedürfnis, für einander zu sorgen, jenseits aller Mythen.

\section{Endnoten}

[1] Der Film setzt chronologisch in der in Spanien umgangssprachlich als die Años del Hambre bezeichneten Periode der Lebensmittelrationierung ein, die vom Ende des Bürgerkrieges (1936-1939) bis, zumindest nach offiziellen Angaben, 1952 reicht.
Abb. 2 Baulücke als Bauplatz „Victoria ¿de quién?" (Quelle: Juan Ortiz Rivas) 
[2] Einer der Verantwortlichen für diese Transformationen ist der Politiker Manuel Fraga Iribarne, von 1962 bis 1969 Minister Francos für Tourismus und Information. 1962 verabschiedete Fraga das Gesetz der touristischen Kompetenzen, um in Spanien die touristische Unterhaltung der Besucher zu fördern, oder, wie der Titel des Gesetzes lautet: „[...] para fomentar en España las excursiones artísticas y de recreo del público extranjero.“ Als Vorläufer dieses Gesetzes gilt das königliche Dekret vom 6. Oktober 1905 von König Alfons XIII, aus dem die Bundeskommission zur Unterstützung des Tourismusgeschäftes in Spanien hervorging.

[3] Zum Zeitpunkt der hier erzählten Ereignisse regierte in Málaga die rechtskonservative Volkspartei PP in Koalition mit den neoliberalen Ciudadanos.

[4] Siehe https://travelerlook.wordpress.com/2017/o5/24/malaga-donde-la-cultura-escapital/ (letzter Zugriff am 1.6.2019); der dazugehörige Werbespot ist verfügbar unter https://www.youtube.com/watch?v=fJKoiJOG1XQ (letzter Zugriff am 15.10.2019).

[5] Enteignung wird hier als räumliche Verdrängung verstanden, wie ,disappropriation ‘ im Englischen.

[6] Dies gilt aufgrund der vorteilhaften Bedingungen, die das sogenannte Bodengesetz oder ,Ley de Régimen del suelo y valoraciones de España', überarbeitet 1996 vom Partido Popular, erzeugt hatte.

[7] In Málagas centro histórico gibt es auf 48,28 Hektar insgesamt 1.319 Gebäude, davon etwa 450 Einheiten für Wohnungen.

[8] Alle Informationen und Daten sind im angehängten Bericht enthalten, den wir mit der Mietergewerkschaft Málaga (Sindicato de Inquilinas de Málaga) erstellt haben: https:// www.dropbox.com/s/io703z7wiavjo6y/Dossier\%20V3.pdf?dl=0. (Letzter Zugriff am 30.6.2019). Daraus werden die Wirkungen der Spekulationen im Immobiliensektor ersichtlich: Vertreibung der Bewohner_innen aus dem Zentrum als Effekt der Mietsteigerungen und Ersetzen der Bewohner_innen durch Besucher_innen.

[9] Unten zu sehen sind die Folgemaßnahmen, die der Nachbarschaftsverband des Zentrums zu diesem Thema durchgeführt hat: Asociación de vecinos. Centro Antiguo de Málaga. http://centroantiguomalaga.com/2018/11/11/la-cuestion-de-las-zas-zonas-acusticamente-saturadas-y-el-levantamiento-de-los-hosteleros-visto-por-los-habitantes-de-la-ciudad/ (Letzter Zugriff am 30.6.2019). Es gibt weitere städtische Kontexte, die man diesbezüglich heranziehen könnte: kulturelles Erbe, Grünflächen, áreas culturales usw.

[10] Es handelt sich hierbei um eine neue Praktik, die unter Tourist_innen populär ist. Schon im Juli 2018, knapp zwei Monate nach Beginn der Sommersaison, teilte La Vanguardia (18.7.2018) mit, dass in Mallorca sechs Touristen, elf seit Januar, durch balconing verunglückt seien.

[11] Um diese Episode der Stadtenteignung und politischen Entmächtigung in Spanien als Entwicklung des Kapitalismus zu betrachten, lohnt es hier, sich Friedrich Engels ‘ Analyse vergleichbarer Prozesse der Ortausgrenzung und Landenteignung von Landarbeitern und Bauern im England des 19. Jahrhunderts zu vergegenwärtigen, bzw. wie diese eigentliche Landentwurzelung in die Bildung und Entstehung einer eigentumslosen und stadtgebundenen Arbeiterklasse überging (vgl. Engels (1972 [1845]: 320).

[12] Ein Wortspiel, das sich sowohl auf die in Málaga geborene Feministin und Politikerin Victoria Kent (Málaga, 1891-1987) als auch auf den Sieg der katholischen Könige über Málaga (1487) bezieht. Victoria Kent wurde in einem der angrenzenden Gebäude, das inzwischen nicht mehr existiert, geboren.

\section{Autor_innen}

Kike España ist Stadtforscher, Architekt und Aktivist und in verschiedenen Antigentrifizierungs- und Right-to-the-City-Bewegungen in Málaga engagiert.

kikespana@gmail.com

Beatriz V. Toscano ist eine in Deutschland lebende spanische Forscherin. Ihr Forschungsschwerpunkt liegt an den Schnittstellen zwischen Stadtplanung und Biopolitiken.

beatrizvtoscano@gmail.com 


\section{Literatur}

Appadurai, Arjun (1986): The Social Life of Things. Commodities in Cultural Perspective. Cambridge: Cambridge University Press.

Bardem, Juan Antonio / Berlanga, Luis Garcia: Bienvenido Mr. Marshall. Madrid: La Novela del Sábado.

Blanchard, Paul / Boëtsch, Gilles (Hg.) (2012): Human Zoos. The invention of the savage. Paris: Actes Su.

Blázquez-Salom, Marcià (2013): More Villas and More Barriers: Gentrification and the Enclosure of Rural Land on Majorca. Méditerranée. In: Revue géographique des pays méditerranéens 120, 25-36.

Bienvenido, Mister Marshall (1953): Luís García Berlanga (Regie), Juan Antonio Bardem/ Luís García Berlanga/ Miguel Mihura (Drehbuch). Spanien: Productoras UNICI.

Cocola-Gant, Agustin (2018): Tourism gentrification. In: Loretta Lees / Martin Phillips (Hg.), Handbook of Gentrification Studies. Cheltenham/Northampton: Edward Elgar Publishing, 281-293.

Engels, Friedrich (1972 [1845]): Die irische Einwanderung. In: Karl Marx / Friedrich Engels, Die Lage der arbeitenden Klasse in England. Werke, Band 2. Berlin: Dietz, 320-324.

Exceltur (2008): Studio sobre el empleo en el sector turístico español. http://www.exceltur. org/wp-content/uploads/2018/o4/ESTUDIO-EMPLEO-SECTORTURISTICO-EXCELTUR.pdf (letzter Zugriff am 30.6.2018).

Fernandez-Savater, Amador (2015): La piel y el teatro. Salir de la política. https://www. eldiario.es/interferencias/piel-teatro-Salir-politica_6_442065819.html (letzter Zugriff am 27.11.2019).

Foucault, Michel (2001): Il faut défendre la société. Cours au Collège de France (1975-1976). https://monoskop.org/images/9/99/Foucault_Michel_Il_faut_defendre_la_societe. pdf (letzter Zugriff am 16.10.2019).

Gonzalez Casanova, Pablo (1965): Internal Colonialism and National Development. In: Studies in Comparative International Development 1/4, 27-37.

Harvey, David (2012): Die urbanen Wurzeln der Finanzkrise. Die Stadt für den antikapitalistischen Kampf zurückgewinnen. In: Supplement der Zeitschrift Sozialismus 2, 7-16.

Henrique Martins, Paulo (2018): Internal Colonialism, Postcolonial Criticism and Social Theory. http://www.journaldumauss.net/?Internal-Colonialism-Postcolonial-Criticismand-Social-Theory (letzter Zugriff am 11.8.2018).

Judd, Dennis / Fainstein, Susan (Hg.) (1999): The Tourist City. New Haven: Yale University Press.

Kirshenblatt-Gimblett, Barbara (1998): Destination Culture. Tourism, Museums and Heritage. Los Angeles: University of California Press.

Lefebvre, Henri (1974): La production de l'espace. In: L‘Homme et la Société. Sociologie de la connaissance marxisme et anthropolgie. Paris: L'Harmattan, 15-32.

Lefebvre, Henri / Regulier, Catherine (2004): Attempt at the Rhythmanalysis of Mediterranean Cities. In: Henri Lefebvre, Rhythmanalysis. London: Continuum.

Marazzi, Christian (2008): Capital and Language. From the New Economy to the War Economy. Semiotext(e). Foreign Agents Series. California: The MIT Press.

Marcinkoski, Christopher (2015): The City that Never Was. New York: Princeton Architectural Press.

Marx, Karl (2003 [1867]): Das Kapital. Kritik der politischen Ökonomie. Stuttgart: Parkland Verlag.

Mignolo, Walter (2003): Historias locales, diseños globales: colonialidad, conocimientos subalternos y pensamiento fronterizo. Madrid: Akal.

Murray, Ivan (2015): Capitalismo y turismo en España. Del „milagro económico“ a la „gran crisis“. Barcelona: Alba Sud.

Russo, Antonio Paolo / Scarnato, Alessandro (2018): Barcelona in Common: A New Urban Regime for the 21st-Century Tourist City? In: Journal of Urban Affairs 40, 455-474.

Schäfer, Robert (2013): Tourismus und Authentizität. Zur gesellschaftlichen Organisation von Außeralltäglichkeit. Bielefeld: transcript.

Scheppe, Wolfgang (2018): Die Grundrente der Kunst und der Ausschluss aus der Stadt Das Exemplarische an Venedigs Singularität. In: ARCH+ 231, 14-31. 
Segovia, Carlos (2017): Aena pone en marcha la mayor operación inmobiliaria de España. In: El Mundo vom 15.11.2017.

Smith, Neil (1990): Uneven Development: Nature, Capital, and the Production of Space. Athens: University of Geogia Press.

Statista (2018): Number of tourists in hotels in Barcelona city from 1990 to 2017. https://www.statista.com/statistics/452060/number-of-tourists-inbarcelona-spain/ (letzter Zugriff 12.10. 2019).

Zukin, Sharon (2008): Consuming Authenticity. From Outposts of Difference to Means of Exclusion. In: Cultural Studies 22/5, 724-748.

Zukin, Sharon (2014): Postcard-perfect: the big business of city branding. https://www. theguardian.com/cities/2014/may/o6/postcard-perfect-the-big-business-of-citybranding (letzter Zugriff am 31.8.2019).

\section{Cities on Sale. Processes of disappropriation and practices of re-appropriation in Spain}

The thriving economic sectors of tourism and real estate speculation in Spain elicit a debate as to the phenomenon of democratic erosion in the European Union. As today's EU strategy pushes Spain to embrace its role as a tourism producer, Europe is being divided into countries that send consumers and those that receive them. The resulting pressure on the tourism industry to come up with new possibilities has led to a substantial transformation in cities, turning them into mere leisure destinations. Seizing this opportunity, the same financial and legal structures that dragged Spain into recession in 2008 are now striking deals with an industry that shares their goal of leveraging the lucrative potential of urban space. With metropolis becoming a commodity, entire city centres are emerging as "life-size theme parks", stripped of all the vital resources needed to make them inhabitable.

The purpose of this paper is to analyse the threat posed by this new way of consuming urban areas to boost tourism. This trend, spurred by the cities' own political and financial elites, could be seen as a new form of intra-territorial colonialism within the European Union. Therefore, the goal of this paper will be to suggest ways of reappropriating urban spaces by resignifying their purpose and cultural conception, drawing on some of Spain's efforts to resist this overthrow such as the city of Malaga's La Casa Invisible (The Invisible House) and the \#MálagaNoSeVende (\#MalagaIsNotForSale) campaign. 
